\title{
Interactions between normal-fluid and superfluid vortex rings in Helium-4
}

\author{
Demosthenes Kivotides \\ Department of Aeronautics, Imperial College London
}

\author{
PACS 67.40.Vs - Vortices and turbulence \\ PACS $47.32 . \mathrm{cb}$ - Vortex interactions \\ PACS 67.30.he - Textures and vortices
}

\begin{abstract}
By employing both vortex-dynamical and self-consistent Navier-Stokes methods of analysis, an investigation of interactions between normal-fluid and superfluid vortex rings in helium- 4 is performed. It is shown that, under the influence of normal-fluid vortices, superfluid vortices grow in size in order to match the size of the latter. In turbulence, this mechanism hints to a tendency towards scale-to-scale energy equipartition in the spectral regimes corresponding to the inertial-range in the normal-fluid. Yet, the analysis shows that the induced superfluid vorticity is not trapped by the cores of the normal-fluid vortices, and, consequently, there is no strong correlation between normal-fluid and induced superfluid vorticity patterns. Hence, there is no significant vortex locking effect.
\end{abstract}

Prologue. - Vortex rings are basic objects of classical fluid dynamics [1]. Indeed, in laminar flow, complicated velocity patterns can be understood in terms of interactions between (much simpler) vortex rings, and in turbulent flow [2], vortex ring-like structures are employed (together with vortex blobs and vortex sheets) as models of classical eddies. Vortex rings are even more important in Bose-Einstein Condensed (BEC) quantum fluids (concisely called superfluids from now on) and, more importantly, in case of helium-4. This is because line-vortices are the only type of topological defects allowed in helium-4, they carry a universal (quantized) circulation, and, due to their topological-defect nature, are stable objects [3]. Moreover, due to their atomicscale size cores, they macroscopically appear as real-life examples of isolated vortex-lines in Euler fluid dynamics. In this way, despite being inviscid, superfluids appear similar to normal-fluids, in the sense that key aspects of vortical viscous flow are also present in an inviscid superfluid flow. These are related to the ability of a classical fluid to freely generate vortical flow modes of vanishingly small energy, a process that appears gapped in superfluids. Indeed, a finite-energy barrier quantum tunnelling process is required for a superfluid in uniform motion to spontaneously decelerate via quantized vortex excitation. In other words, superfluid physics are much richer and more interesting than their inviscid nature implies.

At finite temperatures, BEC quantum fluids include, in addition to superfluid degrees of freedom, a normal-fluid component that corresponds to the (quasi-particle) excitations of the vacuum state, and, in the hydrodynamic regime, obeys (forced) Navier-Stokes dynamics. Normalfluids and vortical superfluids are coupled together via drag (Hall-Vinen) and lift (Iordanskii) forces [4]. Notably, these couplings are only active along quantized vortex contours. Hence, both classical and superfluid vortex rings are present at finite temperature, BEC quantum flows, and their interactions are expected to be key features of superfluid turbulence physics [5-7]. In this Letter, an analysis of normal-fluid/superfluid vortex-ring interactions is attempted. Central points of interest are the morphology of superfluid vorticity induced by normal-fluid rings, and whether any induced superfluid vorticity becomes trapped in regions of intense normalfluid vorticity.

I am going to use two different approaches in my analysis: (a) a purely vortex dynamical formulation, where both normal-fluid and superfluid motions are described via vortex dynamics, and (b) a hybrid methodology, where normal-fluid vortex rings are described by the NavierStokes equation, whilst superfluid vortices by vortex dynamics. The first approach has the advantage of exemplifying the desired physics in a most explicit way, whilst 
the second approach is more physically complete, since it includes the back-reaction from superfluid vortices on normal-fluid vorticity, as well as, a dynamic vortex core structure for the latter. These effects are not captured by vortex filament methods where vortices have fixed vorticity profiles within their cores, and are unconditionally stable. One needs to employ vortex blob methods in order to capture such effects with vortex particle methods.

Vortex dynamics model. - Both fluids are modeled as vortex dynamical systems. In particular, let $\mathbf{X}_{s}\left(\xi_{\mathrm{s}}, \mathrm{t}\right)$ denote vortex positions on the superfluid vortex tangle $\mathcal{L}_{s}$ where $\xi_{s}$ is the arclength parametrization along the vortex loops, and $t$ is time. The evolution equation of $\mathbf{X}_{\mathbf{s}}\left(\xi_{\mathrm{s}}, \mathrm{t}\right)$ is given by $[8]$ :

$\dot{\mathbf{X}}_{s}=\mathbf{V}_{s}+h_{1} \mathbf{X}_{s}^{\prime} \times\left(\mathbf{V}_{n}-\mathbf{V}_{s}\right)+h_{2} \mathbf{X}_{s}^{\prime} \times\left[\mathbf{X}_{s}^{\prime} \times\left(\mathbf{V}_{n}-\mathbf{V}_{s}\right)\right]$ where $\mathbf{V}_{s}$ is the Biot-Savart velocity $\mathbf{V}_{s}\left(\mathbf{X}_{s}\right)=$ $\frac{\kappa}{4 \pi} \int_{\mathcal{L}_{s}} \frac{\left(\mathbf{x}-\mathbf{X}_{s}\right) \times d \mathbf{x}}{\left|\mathbf{x}-\mathbf{X}_{s}\right|^{3}}, \dot{\mathbf{X}}_{s}=\partial \mathbf{X}_{s} / \partial t$ is the superfluid vortex velocity, $\mathbf{X}_{\mathbf{s}}^{\prime}=\partial \mathbf{X}_{\mathbf{s}} / \partial \xi_{\mathrm{s}}$ is the unit tangent vector (indicating the direction of the singular superfluid vorticity), $\mathbf{V}_{\mathbf{n}}$ is the normal-fluid velocity, $\kappa$ is the quantum of circulation, and $h_{1}, h_{2}$ are dimensionless temperature dependent parameters given in terms of fluid properties only [8]: $h_{1}=\frac{\rho_{s} \kappa D_{0}}{D_{0}^{2}+\left(\rho_{n} \kappa+\rho_{s} \kappa\right)^{2}}$, and $h_{2}=\frac{-\rho_{n} \kappa\left(\rho_{n} \kappa+\rho_{s} \kappa\right)-D_{0}^{2}}{D_{0}^{2}+\left(\rho_{n} \kappa+\rho_{s} \kappa\right)^{2}}$, where $D_{0}$ is the drag coefficient of superfluid vortex motion in the normal-fluid [4]. In all calculations performed here, $h_{1}=0.179$ and $h_{2}=-7.98 \times 10^{-2}$.

Similarly, let $\mathbf{X}_{n}\left(\xi_{\mathrm{n}}, \mathrm{t}\right)$ be the vortex positions on the centerlines of the normal-fluid vortex tangle $\mathcal{L}_{n}=$ $\cup_{i=1}^{N_{l}(t)} \mathbf{X}_{n}^{i}\left(\xi_{\mathrm{n}}, \mathrm{t}\right)$, where $N_{l}(t)$ denotes the number of normal-fluid vortex filaments at time $t$. The evolution equation of $\mathbf{X}_{n}\left(\xi_{\mathrm{n}}, \mathrm{t}\right)$ is $\dot{\mathbf{X}}_{n}=\mathbf{V}_{n}\left(\mathbf{X}_{n}\right)$ [9]. Notably, in this vortex dynamical formulation, the back-reaction of $\mathcal{L}_{s}$ on $\mathcal{L}_{n}$ is not included. This is not too critical in the present calculations, since I am going to deal here with relatively dilute $\mathcal{L}_{s}$ tangles. The second (hybrid) formulation based on the Navier-Stokes equation for the normal-fluid allows for two way coupling, and can quantify the strength of back-reaction effects. In a vortex filament method, the key element is the prescription of normal-fluid vorticity profiles within the filaments. I employ here Leonard vortices [10], for which the normal-fluid vorticity $\boldsymbol{\omega}_{n}$ is distributed within the vortex cores in an axisymmetric fashion, without any vortex-line torsion:

$$
\begin{aligned}
\boldsymbol{\omega}_{n}(\mathbf{x}, t)= & \sum_{i} \Gamma \int_{C_{i}} \frac{1}{\sigma_{i}\left(\xi_{n}, t\right)^{3}} \zeta\left(\frac{\left|\mathbf{x}-\mathbf{X}_{n}^{i}\left(\xi_{n}, t\right)\right|}{\sigma_{i}\left(\xi_{n}, t\right)}\right) \\
& \left(\frac{\partial \mathbf{X}_{n}^{i}}{\partial \xi_{n}}+\frac{\mathbf{x}-\mathbf{X}_{n}^{i}\left(\xi_{n}, t\right)}{\sigma_{i}\left(\xi_{n}, t\right)} \frac{\partial \sigma_{i}}{\partial \xi_{n}}\right) d \xi_{n} .
\end{aligned}
$$

Here, $C_{i}$ is the centerline contour of filament $i, \xi_{n}$ is the arclength parametrization, $\Gamma$ is the circulation strength, and $\sigma_{i}\left(\xi_{n}\right)$ is the core radius along a filament. The smoothing kernel $\zeta$ describes the way vorticity spreads around the core centerline. The calculations are done with the Gaussian kernel of reference [11]: $\zeta_{g}\left(\frac{r}{\sigma}\right)=\frac{1}{(2 \pi)^{\frac{3}{2}}} e^{\left[-r^{2} /\left(2 \sigma^{2}\right)\right]}$. Because there can be no helical vortex lines in this model, there can be no axial flow within the cores either. The velocity of the normal fluid at field position $\mathbf{x}$ is given by the Biot-Savart integral

$$
\mathbf{V}_{n}(\mathbf{x})=-\frac{1}{4 \pi} \int \frac{\left(\mathbf{x}-\mathbf{x}^{\prime}\right) \times \omega_{\mathrm{n}}\left(\mathbf{x}^{\prime}\right) \mathrm{d} \mathbf{x}^{\prime}}{\left|\mathbf{x}-\mathbf{x}^{\prime}\right|^{3}},
$$

where, notably, the integral is over all space, and the normal fluid vorticity $\boldsymbol{\omega}_{n}$ is distributed within the normalfluid filament cores as detailed above. One also needs a way of updating the tube radii. Biot-Savart motion changes vortex length, and this in turn results in tube radii dynamics according to the law of filament volume conser, vation during inviscid evolution $\frac{d}{d t}\left(\sigma\left(\mathbf{X}_{n}\right)\left|\frac{\partial \mathbf{X}_{n}}{\partial \xi_{n}}\right| d \xi_{n}\right)=0$, which is applied to each discrete vortex segment at each time step, and it is a direct consequence of the incompressibility of the fluid, and the fact that in Euler dynamics vortex lines move with the fluid velocity. In addition, normal-fluid vortex cores are growing due to the diffusive part of molecular fluctuations (which do not preserve filament volume). This effect is captured with the core spreading method [12]: $\frac{d \sigma^{2}}{d t}=2 \gamma \nu_{n}$, where $\nu_{n}$ in the kinematic viscosity of the normal-fluid, and $\gamma$ is a factor depending on the particular kernel $\zeta_{g}$ employed, and is equal to $\gamma=1$ for my Gaussian choice of kernel. Leonard has also elaborated the numerical analysis of his vortices, which I follow here and discuss in more detail in [9]. Finally, the superfluid vortex dynamics follow a standard treatment, as discussed extensively in other works $[4,8]$.

Interactions between rings of similar size. - I first consider a flow with temperature $T=1.3 \mathrm{~K}$ consisting of one normal-fluid vortex-ring, and a suspension of 25 (randomly positioned and oriented) superfluid vortex-rings of similar radii. This arrangement is a cartoon for the study of interactions between similar size turbulent flow scales in superfluid and normal-fluid. The periodic box has size $l_{b}=0.1 \mathrm{~cm}$, the normalfluid ring radius is $R_{n}=0.025 \mathrm{~cm}$, and the radii of superfluid rings are chosen randomly within the interval $R_{s} \in[0.023,0.031] \mathrm{cm}$. The core of the normal-fluid vortex-ring has radius $\sigma=0.0572 R_{n}$. At $t=0$, the system configuration is shown in fig. 1 (left). The Reynolds number in the normal-flow is $R e=\Gamma / \nu_{n}=5000$, which, for this temperature, corresponds to $\Gamma=11686 \kappa$, where $\kappa=9.97 \times 10^{-4} \mathrm{~cm}^{2} \mathrm{~s}^{-1}$ is the quantum of circulation. This disparity between normal-fluid and superfluid circulations is typical of experimental superfluid turbulent flows. In practice, it means that the normal-fluid ring moves much faster than the superfluid vortices, and in fact the latter behave here, to a good approximation, as passive objects. The superfluid rings are discretized with 

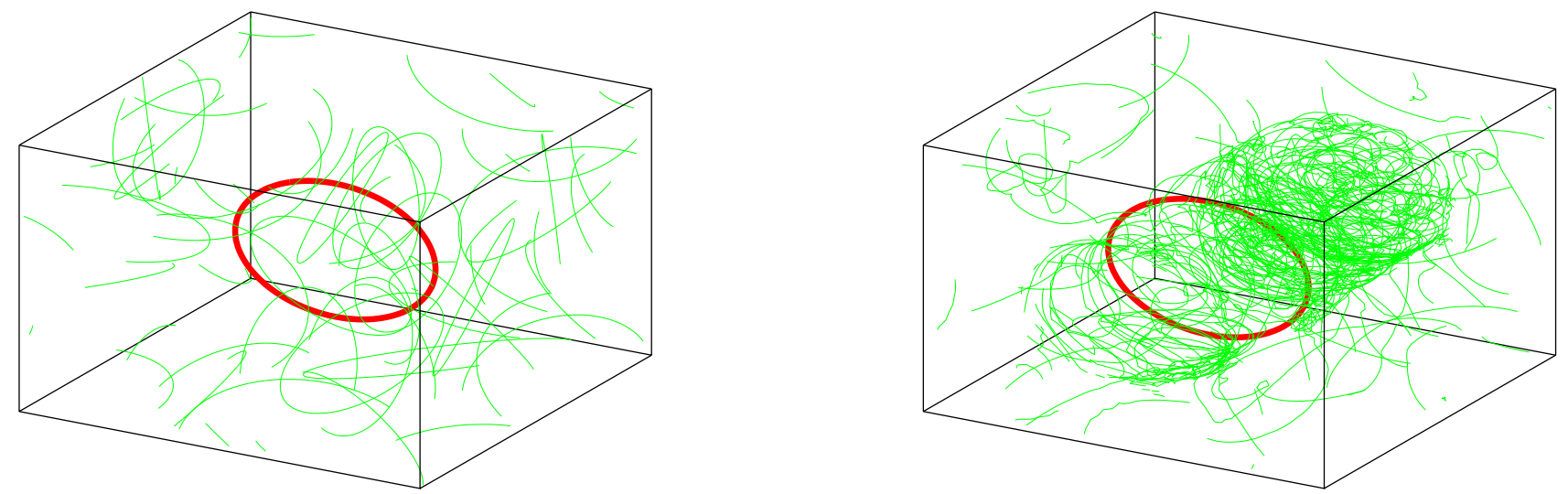

Fig. 1: Left: Normal-fluid vortex ring (thick line) and superfluid vortex rings of similar diameter at $t=0$. Right: Evolution of the initial configuration at $t=1.6527 \times 10^{-3} \mathrm{~s}$. The induced superfluid vorticity is organized into a full "tube" of diameter similar to the size of the normal-fluid vortex-ring.
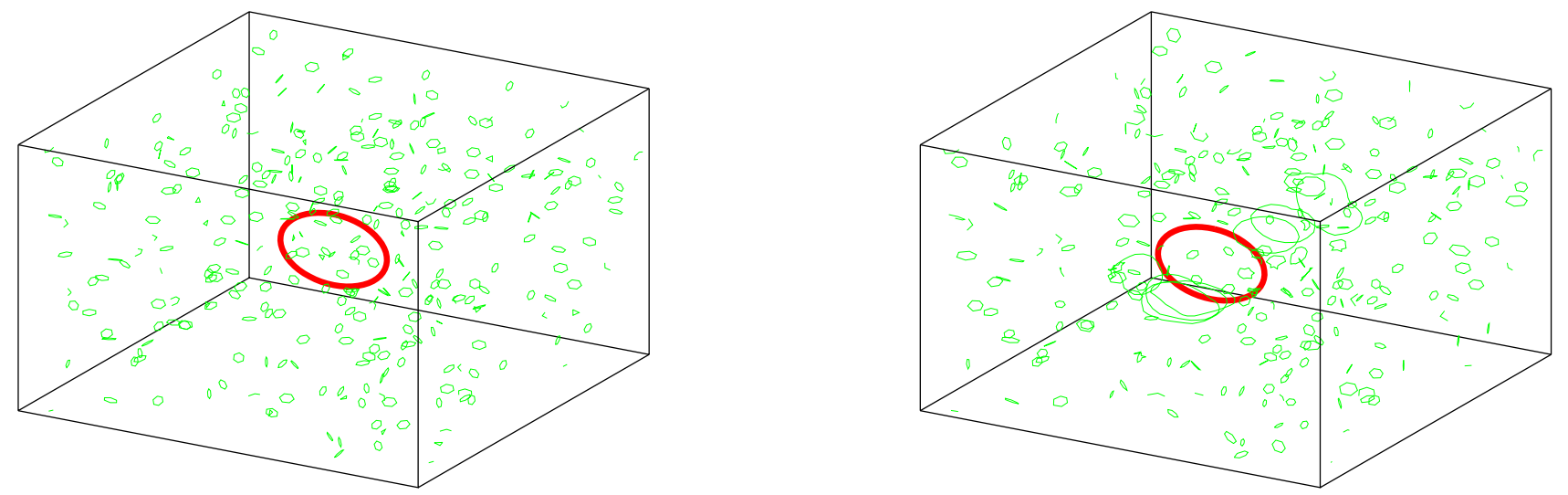

Fig. 2: Left: Normal-fluid vortex ring (thick line) and superfluid vortex rings of much smaller diameter at $t=0$. Right: Evolution of the initial configuration at $t=3.9 \times 10^{-3} \mathrm{~s}$. Small superfluid rings that are "swallowed" by the normal-fluid vortex-ring are blown to a similar to the latter size, but are not trapped by its core.
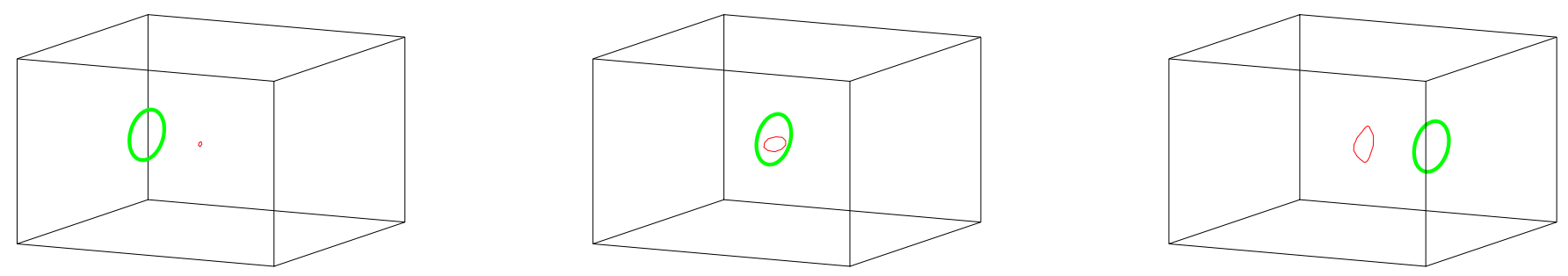

Fig. 3: Left: Normal-fluid vortex ring (thick line) and comoving superfluid vortex ring of much smaller diameter at $t=0$. Center: As the normal-fluid vortex-ring "swallows" the superfluid vortex-ring, the latter's diameter grows $\left(t=5.72 \times 10^{-4} \mathrm{~s}\right)$. Right: The superfluid vortex-ring cannot keep up with the normal-fluid vortex-ring, and it is left behind $\left(t=1.5 \times 10^{-3} \mathrm{~s}\right)$. 


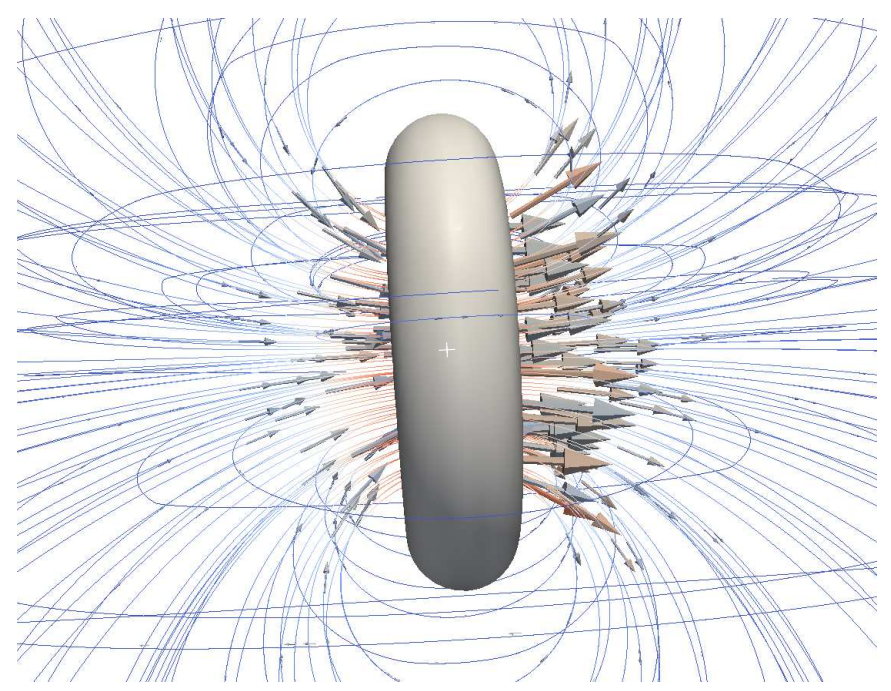

Fig. 4: The core of a normal-fluid vortex ring and its associated streamlines. The vectors indicate the direction of the velocity field along the selected streamlines.

elements of length $l_{b} / 64 \mathrm{~cm}$, and the normal-fluid ring with elements of length $l_{b} / 72 \mathrm{~cm}$. At $t=1.6527 \times 10^{-3} \mathrm{~s}$, the system has evolved to a new state shown in fig. 1 (right). At this time, viscous diffusion has increased the normal-fluid ring core-radius to the value $\sigma=0.125 R_{n}$. The key result is that the normal-fluid ring "swallows" any superfluid vorticity it meets in its path causing it to expand to its own size. This process is accompanied by the superfluid vorticity pushed towards the normal-fluid ring centerline. By itself, this process would lead to the accumulation of superfluid vorticity on the normalfluid centerline supporting the vortex-locking concept. However, the calculation shows that the newly induced superfluid vorticity is not trapped by the normal-fluid vortex core, and instead is left behind forming the tube-like structure of fig. 1 . The physics of this effect is explained later on.

Interactions between rings of disparate sizes. - In order to investigate whether this physical picture extends to interactions between disparate turbulent scales, I insert a normal-fluid ring of radius $R_{n}=0.04 \mathrm{~cm}$ and $R e=\Gamma / \nu_{n}=5000$ within a periodic box of size $l_{b}=0.3 \mathrm{~cm}$, at $T=1.3 \mathrm{~K}$. The core of the ring has size $\sigma=0.1 R_{n}$. This ring interacts with a suspension of 300 superfluid rings of much smaller diameter, i.e., $R_{s} \in[0.08,0.12] R_{n}$. The superfluid rings are discretized with elements of length $l_{b} / 64 \mathrm{~cm}$, and the normal-fluid ring with elements of length $l_{b} / 64 \mathrm{~cm}$. The initial configuration is shown in fig. 2 (left). After a period of time equal to $t=3.9 \times 10^{-3} \mathrm{~s}$, the normal-fluid vortex-core radius has only increased by an imperceptible amount. The system's configuration is shown in fig. 2 (right). The normal-fluid ring had time to interact with a small number of superfluid rings which have grown to

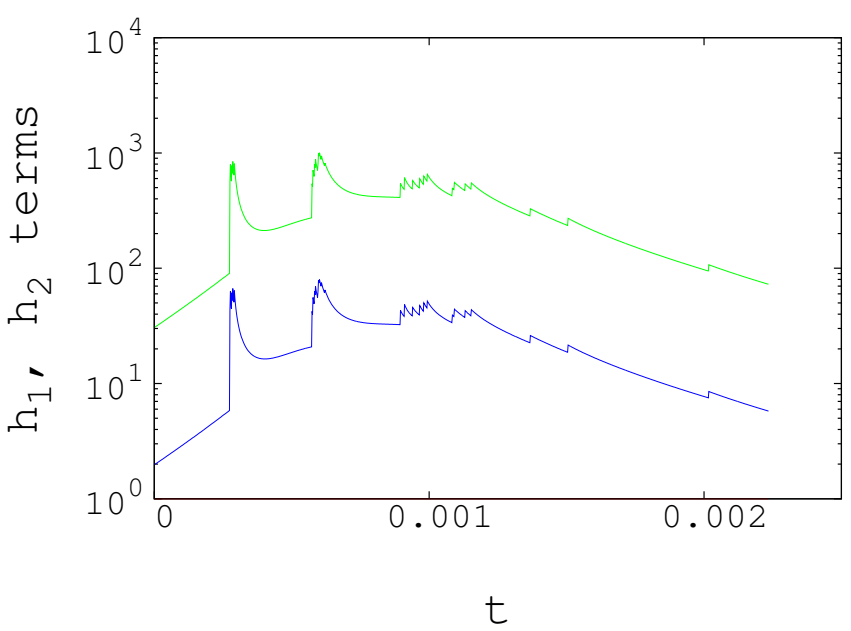

Fig. 5: Time evolution of (scaled with $\left.\left\langle\left|\mathbf{V}_{s}\right|\right\rangle\right)\langle| h_{1} \mathbf{X}_{s}^{\prime} \times\left(\mathbf{V}_{n}-\right.$ $\left.\mathbf{V}_{s}\right)|\rangle$ (upper curve) and $\left\langle\left|h_{2} \mathbf{X}_{s}^{\prime} \times\left[\mathbf{X}_{s}^{\prime} \times\left(\mathbf{V}_{n}-\mathbf{V}_{s}\right)\right]\right|\right\rangle$ (lower curve) terms in the equation for $\dot{\mathbf{X}}_{s}$. The interactions of the superfluid vortex-ring with the normal-fluid vortex-ring are orders of magnitude stronger than its own inertia.

a similar size. This indicates that the effect of ring-ring interactions is to transfer energy from the normal-fluid to the superfluid, driving the energy spectra in both fluids towards equipartition in the range of scales corresponding to the radius of the normal-fluid ring. However, there is not a strong correlation between the actual physical location of the vorticities in the two fluids, since as fig. 2 indicates, the blown-up superfluid rings are not "slaved" to the motion of the normal-fluid ring. Indeed, after a superfluid ring grows during its encounter with the normal-fluid ring, it does show a tendency of following its motion, but this effect cannot match the velocity of the normal-fluid ring which leaves the superfluid ring behind. This finding suggests taking a closer look to the interaction of a single, small superfluid ring with a normal-fluid ring, in order to understand better the mechanism of superfluid vorticity growth.

The mechanism of superfluid ring growth. - In this calculation, the normal-fluid ring described above interacts with a superfluid ring of much smaller diameter, located on a parallel plane (fig. 3, left). The resolutions are kept the same as in the many superfluid rings case above. Both rings move to the right. The normal-fluid ring's core and associated flow streamlines are depicted in fig. 4. Due to the disparate circulation values, the normal-fluid ring catches up with the superfluid one. During their interaction (fig. 3, center) the radius of the superfluid ring grows to a similar to the normal-fluid ring radius. Finally (fig. 3, right), the superfluid ring is left behind. This phenomenology can be understood directly from the equation of motion for $\dot{\mathbf{X}}_{s}$. Due to the disparate circulation values, $\mathbf{V}_{s}$ can be neglected in comparison with $\mathbf{V}_{n}$. Then, taking into account the flow field in fig. 4 


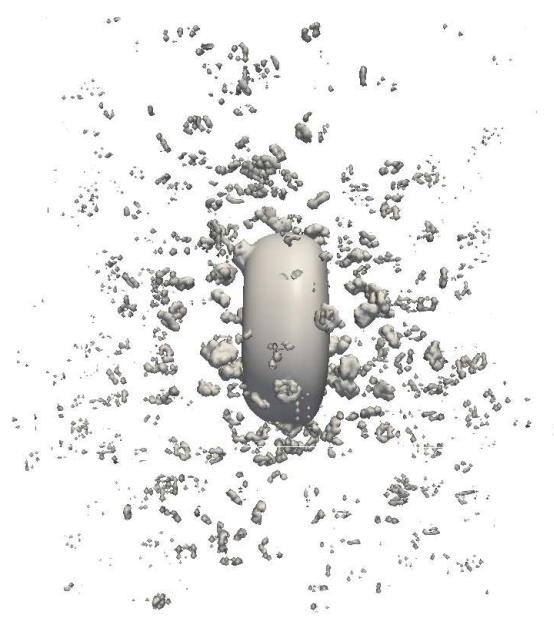

Fig. 6: Initial normal-fluid vorticity isosurface $\left(\left|\boldsymbol{\omega}_{n}\right|=100 \mathrm{~s}^{-1}\right)$ in the Navier-Stokes modeling case. The large central structure is the normal-fluid ring. The numerous "islands" correspond to the normal-fluid vorticity around the superfluid rings, that is induced by the vortex-fluid couplings [13]. These normal-fluid vorticity "halos" around superfluid rings is a good method for their detection in experiments that can measure normal-fluid velocity fields [14].

and the direction of motion of the superfluid ring, it is easy to deduce that the $h_{1}$ term points outwards along the radial superfluid ring direction, hence explaining the growth. On the other hand, the $h_{2}$ term always opposes $\mathbf{V}_{n}$, but since $h_{2}<0$, it corresponds to motion along $\mathbf{V}_{n}$. This explains why, after the normal-fluid ring "swallows" the superfluid ring, there is a tendency of the latter to be trapped by the former. Yet, the normal-fluid ring propagates with velocity of order $\mathbf{V}_{n}$, whilst the superfluid ring moves with velocity $h_{2} \mathbf{V}_{n}$ which is much smaller, since $h_{2}=-7.98 \times 10^{-2}$. This explains why there is no observable vortex locking effect. The dominant role of the normal-fluid/superfluid ring interactions over the latter's inertia is demonstrated in fig. 5. The figure shows the terms $\left\langle\left|h_{1} \mathbf{X}_{s}^{\prime} \times\left(\mathbf{V}_{n}-\mathbf{V}_{s}\right)\right|\right\rangle /\left\langle\left|\mathbf{V}_{s}\right|\right\rangle$ and $\left\langle\left|h_{2} \mathbf{X}_{s}^{\prime} \times\left[\mathbf{X}_{s}^{\prime} \times\left(\mathbf{V}_{n}-\mathbf{V}_{s}\right)\right]\right|\right\rangle /\left\langle\left|\mathbf{V}_{s}\right|\right\rangle$ versus time. The averages are formed over the superfluid ring contour. The term responsible for superfluid vortex ring growth dominates the term dragging the vortex, and both of them dominate the superfluid ring's inertia. Their effect is to induce superfluid vorticity at the scale of normal fluid vorticity without locking the two vorticities together.

Hybrid Navier-Stokes/Vortex-dynamics model. - I switch here to a fully coupled computation, where the normal-fluid ring obeys Navier-Stokes physics, i.e., its evolution satisfies the the mass equation $\nabla \cdot \mathbf{V}_{n}=0$, and the momentum equation

$$
\frac{\partial \mathbf{V}_{n}(\mathbf{x}, t)}{\partial t}+\nabla\left(\frac{p}{\rho_{n}+\rho_{s}}+\frac{\mathbf{V}_{n} \cdot \mathbf{V}_{n}}{2}\right)-\mathbf{V}_{n} \times\left(\nabla \times \mathbf{V}_{n}\right)-
$$

Fig. 7: Normal-fluid vorticity isosurface $\left(\left|\boldsymbol{\omega}_{n}\right|=200 \mathrm{~s}^{-1}\right)$ at $t=7.452 \times 10^{-2} \mathrm{~s}$ (Navier-Stokes modeling case). In agreement with vortex methods, strongly affected superfluid rings grow to (approximately) the normal-fluid ring size. Moreover, deformations of normal-fluid ring core structure due to collisions with superfluid rings are also observed.

$$
\begin{aligned}
& \frac{\mu}{\rho_{n}} \nabla^{2} \mathbf{V}_{n}-\kappa \int_{\mathcal{L}} d\left|\mathbf{X}_{\mathcal{L}}\right|\left[\mathbf{X}_{\mathcal{L}}^{\prime} \times\left(\mathbf{V}_{n}-\dot{\mathbf{X}}_{\mathcal{L}}\right)\right] \delta^{3}\left(\mathbf{x}-\mathbf{X}_{\mathcal{L}}\right)- \\
& \frac{D_{0}}{\rho_{n}} \int_{\mathcal{L}} d\left|\mathbf{X}_{\mathcal{L}}\right|\left\{\mathbf{X}_{\mathcal{L}}^{\prime} \times\left[\mathbf{X}_{\mathcal{L}}^{\prime} \times\left(\mathbf{V}_{n}-\dot{\mathbf{X}}_{\mathcal{L}}\right)\right]\right\} \delta^{3}\left(\mathbf{x}-\mathbf{X}_{\mathcal{L}}\right)=0 .
\end{aligned}
$$

Here, $p$ is the pressure field, and $\mu$ the normal-fluid viscosity. From start to end, we have the inertia, potential ("Bernoulli-group"), vortex, viscous, lift and drag (mutual-friction) forces [4]. The last two forces signify the coupling of normal-fluid with superfluid vortices, and will be collectively called "vortex couplings". Navier-Stokes physics can overcome shortcomings of the vortexdynamical formulation by including the back-reaction (i.e., vortex couplings) from the superfluid vortices on the normal-fluid ring, as well as, a dynamic vortex core. As initial condition, I introduce a normal-fluid ring of radius $R_{n}=0.04 \mathrm{~cm}$ and $R e=\Gamma / \nu_{n}=500$ within a periodic box of size $l_{b}=0.3 \mathrm{~cm}$, at $T=1.3 \mathrm{~K}$. The core of the ring has size $\sigma=0.25 R_{n}$. This ring interacts with a suspension of 600 superfluid rings of much smaller diameter, i.e., $R_{s} \in[0.08,0.12] R_{n}$. The initial configuration is similar to the one shown in fig. 2 (left). The Navier-Stokes equation is solved on an $128^{3}$ grid. The superfluid rings are discretized with elements of length $l_{b} / 64 \mathrm{~cm}$. In order to initialize the normal-fluid flow field, the initial Leonard vortex was discretized with elements of length $l_{b} / 512 \mathrm{~cm}$. The time step is chosen so that viscous diffusion processes are resolved, hence $\Delta t=8.65 \times 10^{-5} \mathrm{~s}$. The particular Reynolds number and $\sigma / R_{n}$ values are chosen in order to avoid the well known instabilities in the propagation of classical vortex rings [15-17], which would only obfuscate the present conclusions.

The initial conditions are shown on fig. 6. The large 
structure is the normal-fluid ring, and the cloud of smaller ones is comprised by the normal-fluid vorticity "halos" of superfluid vorticity first discovered in [13]. The "halos" correspond to normal-fluid flow induced by vortex couplings at the locations of superfluid vorticity. Modern experimental methods [14] that measure normal-fluid velocity fields can exploit these halos in order to detect superfluid rings in a non-invasive way. As fig. 6 shows, the initial superfluid vorticity appears at a much smaller scale than the normal-fluid one. As the system evolves, the physics resemble those in the vortex-dynamical case. Indeed, fig. 7 shows vorticity configuration in the normal-fluid at $t=7.45 \times 10^{-2} \mathrm{~s}$. The large normalfluid ring structure is discernible together with thinner structures that correspond to blown-up superfluid vortex rings that have been left behind by the swiftly moving normal-fluid ring. Thus, the main physical conclusions are not altered in the more powerful modeling case. One difference with previous results is that the normal-fluid ring core is dynamic, hence it is deformed by its collisions with the superfluid rings (fig. 7). However, due to the (relatively) weak circulation of the superfluid rings, such collisions do not appear to be dynamically important.

Epilogue. - Using an array of physical models and calculations, I have shown that the major effect of normal-fluid vortex rings on superfluid ones is an energy transfer that blows-up the latter to the size of the former. During such processes, two fluid interactions are orders of magnitude stronger than inertial effects in the superfluid. Thus, it is expected that, as a result of these interactions, the energy content of spectrum scales in the superfluid that correspond to those in the normal-fluid ought to grow. This process is not accompanied by the locking of vorticities in the two fluids. This implies that the coherent superfluid vortex bundles studied in [18] must be put in the system "by experimental design", and are not emergent structures of superfluid turbulence. For the case of fully developed turbulence at finite temperature superfluid helium-4, the implication is that, at steady state, homogeneous, isotropic turbulence, the energies in the two fluids are expected to reach an equilibrium with similar but not necessarily equal levels. The actual energy level ratios would be universal and a function of the particular scale in the spectrum. Testing this conjecture for realistic Reynolds number values would require large-scale numerical calculations that could be perfomed in the near future.

In another important context, I mentioned above that the choice of the Navier-Stokes vortex ring parameters was dictated by the need to avoid any high $R e$ number normal-fluid ring instabilities. In this respect, the observation of [19] of stable normal-fluid vortex rings of $10^{4} \leq R e \leq 5 \times 10^{4}$ (i.e., above the classical stability limit) can only be attributed to the effects of superfluid vorticity via a kind of damping mechanism which could be revealed in the future by employing the physical model of $[4]$.

\section{REFERENCES}

[1] Aкhmetov D.G., Vortex Rings (Springer, Berlin) 2009.

[2] Davidson P.A., turbulence (Cambridge University Press, Cambridge) 2004, p. 203.

[3] Donnelly R.J., Quantized Vortices in Helium II (Cambridge University Press, Cambridge) 2005.

[4] Kivotides D., Phys. Fluids, 26 (2014) 105105.

[5] Borner H., Schmeling T. \& Schmidt D.W., Phys. Flu$i d s, 26$ (1983) 1410.

[6] Stamm G., Bielert F., Fiszdon W. \& Piechna, J., Physica B, 193 (1994) 188.

[7] Stamm G., Bielert F., Fiszdon W. \& Piechna, J., Physica B, 194-196 (1994) 589.

[8] Kivotides D., J. Fluid Mech., 668 (2011) 58.

[9] Kivotides D. and Wilkin S.L., J. Low Temp. Phys., 156 (2009) 163.

[10] Leonard A., Phys. Fluids, 6 (1994) 765.

[11] Leonard A. and Winckelmans G.S., J. Comp. Phys., 2 (1993) 247.

[12] Leonard A., J. Comp. Phys., 37 (1980) 289.

[13] Kivotides D., Barenghi C.F. and Samuels D.C., Science, 290 (2000) 777.

[14] Guo W., Wright J.D., Cahn S.B., Nikkel J.A. and McKinsey D.N., Phys. Rev. Lett., 102 (2009) 235301.

[15] Bergdorf M., Koumoutsakos P. and Leonard A., J. Fluid Mech., 581 (2007) 495.

[16] Shariff K. and Leonard A., Annu. Rev. Fluid Mech. 24 (1992) 235.

[17] Shariff K., Verzicco, R. and Orlandi P., J. Fluid Mech., 279 (1994) 351.

[18] Wacks D.H., Baggaley A.W. \& Barenghi C.F., Phys. Fluids, 26 (2014) 027102.

[19] Murakami M., Hanada M. \& Yamazaki T., Jpn. J. Appl. Phys. Suppl., 26 (1987) 107. 